\title{
An efficient space-time based simulation approach of wind velocity field with embedded conditional interpolation for unevenly spaced locations
}

\author{
Liuliu Peng ${ }^{\mathrm{a}}$, Guoqing Huang ${ }^{* a}$, Ahsan Kareem ${ }^{\mathrm{b}}$, Yongle Li ${ }^{\mathrm{a}}$ \\ ${ }^{a}$ Research Center for Wind Engineering, School of Civil Engineering, Southwest \\ Jiaotong University, Chengdu, Sichuan 610031 China \\ ${ }^{\mathrm{b}}$ NatHaz Modeling Laboratory, Department of Civil Engineering and Geological \\ Sciences, University of Notre Dame, Notre Dame, IN 46556 USA
}

\section{ABSTRACT}

The classical spectral representation method (SRM) has been extensively used in the simulation of multivariate stationary Gaussian random processes. Due to the application of fast Fourier transform (FFT), the simulation is usually efficient. However, for processes with a large number of simulation points, it becomes necessary to enhance the simulation efficiency. One example is the wind velocity field along a large-span bridge, where hundreds of wind velocity fluctuations are required. In the case of bridges built over a homogeneous terrain such as coastal area or flat plain, the wind velocity field can be modeled as a multivariate homogeneous random process i.e., the auto power spectral densities (PSDs) at evenly-spaced simulation points are same and the cross PSD is a function of separation distance between two simulation points. Furthermore, in some applications, additional simulation points need to be included to a set of uniformly distributed points in order to make the wind velocity field consistent to the structural dynamic analysis requirements.

In this paper, a hybrid approach of space-time random-field based SRM and proper orthogonal decomposition (POD)-based interpolation is developed for simulating the above wind velocity process. In this approach, the random-field based SRM is used to simulate the multivariate homogeneous random process composed of a set of uniformly distributed simulation locations while POD-based interpolation is used to conditionally generate the wind velocities at a few unevenly distributed points using the previously simulated wind velocities. The idea of the former is based on transforming the simulation of the homogeneous random process into that of the corresponding space-time random field where the phase angle is assumed to be zero and the coherence function must be an even function in terms of separation distance. Through this procedure, customary requirement for spectral matrix decomposition is eliminated and application of two dimensional FFT can improve the simulation efficiency dramatically. The shortcomings of this method include a slight approximation regarding the simulated sample and the non-ergodicity for the correlation function. The numerical example of a homogeneous wind velocity field along a bridge deck shows that the proposed random field-based method is very efficient in terms of accuracy and efficiency when the number of simulation locations is large and the POD-based interpolation also has good performance. 
Keywords: Stationary; Multivariate Random Processes; Wind Velocity Field; Homogeneous; Simulation; Space-time Random Field; Two-dimensional Fast Fourier Transform; Ergodicity; Conditional Interpolation; Proper Orthogonal Decomposition

\section{Introduction}

The frequency domain based analysis approach is generally preferred in engineering practice, but Monte Carlo simulation is still widely used in case where nonlinearity, system stochasticity, and other related problems exist. For example, Monte Carlo simulation approach is more convenient for considering the structural and aerodynamic nonlinearities in the wind-induced dynamic analysis of long-span bridges and tall buildings. Also Monte Carlo simulation often serves as a benchmark to evaluate accuracy of other approaches such as the frequency domain based approaches.

One of most important components in Monte Carlo simulation is to generate sample functions of stochastic excitations, such as ground motions, wind and wave fluctuations. Based on spectral representation theorem, the spectral representation method (SRM) is widely used in the simulation of sample functions of stochastic processes and fields due to its expediency (e.g., Shinozuka and Jan 1972; Spanos and Mignolet 1989; Li and Kareem 1993; Grigoriu 2000). The computational efficiency of SRM-based simulation of stationary Gaussian processes and fields can be considerably enhanced by the way of introducing the fast Fourier transform (FFT) (Yang 1972). Deodatis (1996) summarized the classical SRM-based simulation for multivariate stationary Gaussian processes and Shinozuka and Deodatis (1996) reviewed the simulation of stationary Gaussian fields by SRM.

Despite the successful application of SRM in generating sample functions for stationary Gaussian processes, there is room to improve its efficiency, especially for large structures such as tall buildings and long-span bridges where a very large number of simulation points could be required. One example is the wind-induced buffeting analysis of large-span bridges, where hundreds of wind velocity fluctuations need to be generated to better represent the wind velocity field along the length of the deck. Also, perhaps around a thousand wind velocity locations are needed for the analysis of wind-vehicle-bridge systems when a high-speed train passes a bridge. For the bridge built over a homogeneous terrain such as coastal area or flat plain, the wind velocity field can be modeled as a multivariate homogeneous random process, i.e., the auto power spectral densities (PSDs) at evenly-spaced simulation points are same and the cross PSD is a function of separation distance between two simulation points. In some applications, additional simulation points need to be included to a set of simulations at uniformly distributed points in order to make the wind velocity field consistent to the need of structural dynamic analysis, which adds to computational demand.

The simulation of aforementioned multivariate processes requires significant computational time and storage memory if the classical SRM is adopted. For instance, the double-index frequency technique introduced by Deodatis (1996) can generate ergodic sample functions. However, this treatment needs decomposition of a very large spectral matrix when the number of simulation points is large. Besides, the 
generated ergodic sample will have a very long period since the period of samples has a linear relation with the number of the simulation points, which reduces the simulation efficiency and increases computational demand for the ensuing response analysis. To enhance the simulation efficiency or save the memory requirements in the generation of random processes, the common practice is to reduce the computational demand on the Cholesky decomposition of spectral matrix in that the decomposition of large matrix consumes significant computer resources. Yang et al. (1997) and Cao et al. (2000) proposed closed-form formula for wind field simulation to avoid Cholesky decomposition of the spectral matrix. This method is effective only if the coherence function of the random process follows a real exponential function. However, many studies show that this simple model for winds may not be appropriate, and several more meaningful non-exponential models have been introduced (e.g., Kareem 1987; Krenk 1996; Dyrbye and Hansen 1997; Mann 1998). In addition, the closed-form formula technique is only valid for the multivariate homogeneous random process with evenly distributed simulation points. Ding et al. (2011) derived an efficient scheme to decrease the computational amount of spectral matrix decomposition, where the decomposition of the spectral matrix is performed on a set of single-index frequencies. Huang et al. (2013) developed a new form of Cholesky decomposition to improve the decomposition efficiency of the spectral matrix by separating the phase from the spectral matrix. Apart from the widely-used Cholesky decomposition, the proper orthogonal decomposition (POD), or eigenvector decomposition has been used in the simulation of multicorrelated stationary random processes or fields in both the time and frequency domains (e.g., Li and Kareem 1993; Yamazaki and Shinozuka 1990; Di Paola and Gullo 2001; Chen and Letchford 2005; Chen and Kareem 2005; Liu et al. 2011). In addition, POD has also been widely employed to simplify the simulation of wind velocity fields such as random wind fields with varying PSDs at different locations, complex wind fields with three correlated turbulence components along a linear domain and complex wind fields considering three turbulent components in a spatial domain, where the closed-form solution for the eigenvalue and eigenfunction of the principal turbulence was derived for exponential coherence function (Carassale and Solari 2002 and 2006; Tubino and Solari 2005). The core of utilizing POD is that the simulation may be carried out on only a few lower modes which contain most of the energy. In this case, the simulation efficiency can be improved. However, the truncation of higher modes may cause noticeable errors if local structural response is of interest (Chen and Kareem 2005). Besides, POD may fall short in very broad-band processes or fields such as the white noise. It should be noted that both the Cholesky and POD decompositions can be unified under the concept of stochastic decomposition approach proposed by $\mathrm{Li}$ and Kareem (1993 and 1995).

On the other hand, the application of FFT is a good option to improve the simulation efficiency for wind velocity processes. As is well known, the two-dimensional (2D) FFT algorithm can significantly expedite the simulation of Gaussian stationary random field (e.g., Zerva 1992; Shinozuka and Deodatis 1996). Hence, if the multivariate wind velocity process can be transformed to a space-time 
random field, 2D FFT algorithm could be introduced and simulation efficiency may be significantly enhanced. Although the space-time random field has been applied to simulate stochastic excitations such as seismic ground motions and wind fields (e.g., Shinozuka 1972; Zerva and Zervas 2002; Benowitz and Deodatis 2013), a systematic treatment of the subject has thus far not been addressed.

To efficiently simulate the wind velocity field along the bridge deck with a large number of points, a hybrid simulation approach will be developed in this study. Primarily, a multivariate homogeneous random process associated with a set of uniformly distributed simulation points can be simulated via the random field-based simulation method. Then, the wind velocities at a few unevenly distributed points can be stochastically interpolated from the simulated wind velocities. Among many interpolation schemes, the POD is a powerful one which has been widely used in stochastic mechanics and wind engineering including the compression and reconstruction of wind pressures (e.g., Bienkiewicz et al. 1993; Tamura et al. 1999; Chen and Kareem 2005) and the factorization of decomposed spectra of evolutionary PSD (Huang 2015). This study utilizes a POD-based interpolation scheme.

This paper is organized as follows. First, the characteristics of the wind velocity field with a large number of simulation points are discussed briefly. Then an efficient simulation approach relying on the random field-based SRM and POD-based interpolation is introduced to generate the sample function of this wind velocity field. Especially, the applicability, accuracy, ergodicity and efficiency of the random field-based SRM are examined. Furthermore, a numerical example is used to demonstrate the effectiveness of the proposed approach. In the end, some concluding remarks will be given.

\section{Wind velocity field}

Let $\left[p_{1}^{0}(t), p_{2}^{0}(t), \cdots, p_{l}^{0}(t)\right]^{T}$ be a zero-mean $l$-variate wind velocity field along a bridge deck over a homogeneous terrain. The underlying correlation function and associated spectral matrices are given by

$$
\begin{gathered}
\mathbf{R}^{0}(\tau)=\left[\begin{array}{cccc}
R_{11}^{0}(\tau) & R_{12}^{0}(\tau) & \cdots & R_{1 l}^{0}(\tau) \\
R_{21}^{0}(\tau) & R_{22}^{0}(\tau) & \cdots & R_{2 l}^{0}(\tau) \\
\vdots & \vdots & \ddots & \vdots \\
R_{l 1}^{0}(\tau) & R_{l 2}^{0}(\tau) & \cdots & R_{l l}^{0}(\tau)
\end{array}\right] \\
\mathbf{S}^{0}(\omega)=\left[\begin{array}{cccc}
S_{11}^{0}(\omega) & S_{12}^{0}(\omega) & \cdots & S_{1 l}^{0}(\omega) \\
S_{21}^{0}(\omega) & S_{22}^{0}(\omega) & \cdots & S_{2 l}^{0}(\omega) \\
\vdots & \vdots & \ddots & \vdots \\
S_{l 1}^{0}(\omega) & S_{l 2}^{0}(\omega) & \cdots & S_{l l}^{0}(\omega)
\end{array}\right]
\end{gathered}
$$

where $\tau$ and $\omega$ are the time lag and circular frequency, respectively; $R_{j k}^{0}(\tau)$ and

$S_{j k}^{0}(\omega) \quad(j, k=1,2, \cdots, l)$ are the cross correlation function and cross PSD (XPSD), 
respectively. For $j=k$, these reduce to the auto correlation function and auto PSD. $R_{j k}^{0}(\tau)$ and $S_{j k}^{0}(\omega)$ are related to each other through the Wiener-Khintchine transformation

$$
\begin{gathered}
R_{j k}^{0}(\tau)=\int_{-\infty}^{\infty} S_{j k}^{0}(\omega) \mathrm{e}^{i \omega \tau} d \omega \\
S_{j k}^{0}(\omega)=\frac{1}{2 \pi} \int_{-\infty}^{\infty} R_{j k}^{0}(\tau) \mathrm{e}^{-i \omega \tau} d \tau
\end{gathered}
$$

It is well known that matrix $\mathbf{S}^{0}(\omega)$ is Hermitian and nonnegative definite. Since the terrain is homogeneous, one has $S_{j j}^{0}(\omega)=S_{k k}^{0}(\omega)$. The XPSD between any two component processes can be given as

$$
S_{j k}^{0}(\omega)=S_{j j}^{0}(\omega) \gamma_{j k}(\omega) e^{i \theta_{j k}(\omega)}, j \neq k
$$

where $\gamma_{j k}(\omega)=\left|S_{j k}^{0}(\omega)\right| / S_{j j}^{0}(\omega)$ is the coherence function between component processes $p_{j}^{0}(t) \quad$ and $p_{k}^{0}(t) ; \quad \theta_{j k}(\omega)=\tan ^{-1}\left(\operatorname{Im}\left[S_{j k}^{0}(\omega)\right] / \operatorname{Re}\left[S_{j k}^{0}(\omega)\right]\right) \quad$ is the corresponding phase angle; Im and Re denote the imaginary and real parts, respectively. According to Di Paola (1998), $\theta_{j k}(\omega)$ can be expressed in the form

$$
\theta_{j k}(\omega)=\frac{-\omega\left(z_{j}-z_{k}\right)}{v_{\text {app }}^{j k}}
$$

where $z_{j}$ is the height of wind velocity component $p_{j}^{0}(t) ; v_{\mathrm{app}}^{j k}$ is the apparent velocity of waves. This equation is a measure of the wave passage delay due to the apparent velocity of wave. From Eq. (6), it can be seen that the phase angle will be zero when all the simulation points are lying on a horizontal line. Correspondingly, the complex XPSD will reduce to a real one.

Assume that the majority of simulation points of the wind velocity field are evenly distributed and a few points cannot be included in this set of evenly distributed points. Then this wind velocity field is regarded to be a $l$-variate homogeneous wind velocity field where $n(n<l)$ simulation points are evenly distributed and additional $(l-n)$ simulation points are unevenly distributed. An example is shown in Fig. 1 where the homogeneous wind velocity field of 304 points along the bridge deck is composed of wind velocities of 301 evenly distributed points and the velocities at unevenly distributed point $\mathrm{A}, \mathrm{B}$ and $\mathrm{C}$. The wind velocity field will be generated by a hybrid simulation approach, as discussed in the next section. 
In this section, a simulation approach for the wind velocity field is introduced. First, the random field-based method is adopted to simulate a multivariate homogeneous random process composed of a set of uniformly distributed simulation locations. Second, a POD-based interpolation is used to conditionally generate the wind velocities at a few unevenly distributed points from the simulated wind velocities.

\subsection{Random field-based spectral representation method}

Let $f^{0}(x, t)$ be a zero-mean univariate $2 \mathrm{D}$ homogeneous and continuous space-time random field. The 2D space-time correlation function $R_{f f}^{0}(\xi, \tau)=E\left[f^{0}(x, t) f^{0}(x+\xi, t+\tau)\right]$ ( $E$ is expectation operator) and PSD function $S_{f f}^{0}(\kappa, \omega)$ of this space-time random field are related by

$$
R_{f f}^{0}(\xi, \tau)=\int_{-\infty}^{\infty} \int_{-\infty}^{\infty} S_{f f}^{0}(\kappa, \omega) e^{i(\kappa \xi+\omega \tau)} d \kappa d \omega
$$

$$
S_{f f}^{0}(\kappa, \omega)=\frac{1}{(2 \pi)^{2}} \int_{-\infty}^{\infty} \int_{-\infty}^{\infty} R_{f f}^{0}(\xi, \tau) e^{-i(\kappa \xi+\omega \tau)} d \xi d \tau
$$

where $\xi$ is separation distance and $\kappa$ is the corresponding wave number. In aforementioned equations, the 2D PSD $S_{f f}^{0}(\kappa, \omega)$ is a real and nonnegative function of wave number and frequency (Shinozuka and Deodatis 1996). Also both of space-time correlation function and 2D PSD are symmetric with respect to the origin, that is,

$$
R_{f f}^{0}(\xi, \tau)=R_{f f}^{0}(-\xi,-\tau)
$$

$$
S_{f f}^{0}(\kappa, \omega)=S_{f f}^{0}(-\kappa,-\omega)
$$

Furthermore, the XPSD $S_{f}^{0}(\xi, \omega)$ between $f^{0}(x, t)$ and $f^{0}(x+\xi, t)$ is associated with the corresponding 2D PSD $S_{f f}^{0}(\kappa, \omega)$ through the Wiener-Khintchine transformation

$$
\begin{gathered}
S_{f}^{0}(\xi, \omega)=\int_{-\infty}^{\infty} S_{f f}^{0}(\kappa, \omega) e^{i \kappa \xi} d \kappa \\
S_{f f}^{0}(\kappa, \omega)=\frac{1}{2 \pi} \int_{-\infty}^{\infty} S_{f}^{0}(\xi, \omega) e^{-i \kappa \xi} d \xi
\end{gathered}
$$

215 To make the transformation in Eq. (12) valid, the XPSD $S_{f}^{0}(\xi, \omega)$ has to be a real 
and an even function in terms of the separation distance $\xi$ in order to remain a real and nonnegative 2D PSD (Shinozuka 1972).

Suppose the multivariate homogeneous wind velocity process $\left[p_{1}^{0}(t), p_{2}^{0}(t), \cdots, p_{n}^{0}(t)\right]^{T}$ is a collection of component processes, evenly spaced along locations $x_{1}, x_{2}, \cdots, x_{n}$ on a straight line. Accordingly, this homogeneous process can be regarded as the discretized space-time random field at locations $x_{1}, x_{2}, \cdots, x_{n}$ along the space axis, i.e., $\left[f^{0}\left(x_{1}, t\right), f^{0}\left(x_{2}, t\right), \cdots, f^{0}\left(x_{n}, t\right)\right]^{T}$ and simulation of a homogeneous random process with evenly-spaced simulation points can be transformed to that of the corresponding random field.

Before conducting the simulation, XPSD of the transformed wind velocity field should be obtained from Eq. (5). For the wind velocity field, the phase angle may be assumed to be zero, especially in the case of all simulation points being on a horizontal bridge deck. Consequently, the XPSD of the transformed wind velocity field becomes a real function. If the coherence function in Eq. (5) is also an even function in terms of separation distance $\xi$, the transformed condition will be valid, which permits the simulation of a multivariate homogeneous wind velocity process by the corresponding space-time random field. At this juncture, the following equation holds based on Eq. (12)

$$
S_{f f}^{0}(\kappa, \omega)=S_{f f}^{0}(-\kappa, \omega)=S_{f f}^{0}(\kappa,-\omega)=S_{f f}^{0}(-\kappa,-\omega)
$$

Hence, the transformed random field reduces to be quadrant symmetric.

\subsubsection{Simulation scheme}

In the following, the distinction will be made for the random process $\left[p_{1}^{0}(t), p_{2}^{0}(t), \cdots, p_{n}^{0}(t)\right]^{T}$ and random field $f^{0}(x, t)$, and their corresponding simulations $\left[p_{1}(t), p_{2}(t), \cdots, p_{n}(t)\right]^{T}$ and $f(x, t)$.

If $S_{f}^{0}(\xi, \omega)$ is a real and even function in terms of separation distance, the transformed random field from the homogeneous random process reduces to a quadrant-symmetric homogeneous random field. Hence, the simulation formula can be given as follows

$$
\begin{aligned}
f(x, t) & =\sqrt{2} \sum_{n_{1}=0}^{N_{1}-1} \sum_{n_{2}=0}^{N_{2}-1}\left\{\sqrt { 2 S _ { f f } ^ { 0 } ( \kappa _ { n _ { 1 } } , \omega _ { n _ { 2 } } ) \Delta \kappa \Delta \omega } \left[\cos \left(\kappa_{n_{1}} x+\omega_{n_{2}} t+\Phi_{n_{1} n_{2}}^{(1)}\right)\right.\right. \\
& \left.\left.+\cos \left(\kappa_{n_{1}} x-\omega_{n_{2}} t+\Phi_{n_{1} n_{2}}^{(2)}\right)\right]\right\}
\end{aligned}
$$

where $\kappa_{n_{1}}=\left(n_{1}+\varepsilon\right) \Delta \kappa, \Delta \kappa=\kappa_{u} / N_{1}$ and $n_{1}=0,1, \cdots, N_{1}-1 ; \quad \omega_{n_{2}}=\left(n_{2}+\varepsilon\right) \Delta \omega$, 
$\Delta \omega=\omega_{u} / N_{2}$ and $n_{2}=0,1, \cdots, N_{2}-1 ; \varepsilon$ is a fraction $1 / m$ and $m$ is integer

larger than $1 ; \kappa_{u}$ and $\omega_{u}$ are the cut-off wave number and frequency, respectively; $\Phi_{n_{1} n_{2}}^{(1)}$ and $\Phi_{n_{1} n_{2}}^{(2)}$ are two independent random phase angles uniformly distributed in the range $[0,2 \pi]$. By substituting these random phase angles with their respective $i$-th realizations $\phi_{n_{1} n_{2}}^{(1)(i)}$ and $\phi_{n_{1} n_{2}}^{(2)(i)}$, a sample function $f^{(i)}(x, t)$ of the transformed random field can be obtained. Note that during the sample function simulation, the space increment $\Delta x$ and time increment $\Delta t$ separating the generated values of $f^{(i)}(x, t)$ must satisfy the requirement: $\Delta x \leq 2 \pi /\left(2 \kappa_{u}\right)$ and $\Delta t \leq 2 \pi /\left(2 \omega_{u}\right)$ in order to avoid aliasing according to the sampling theorem.

If $\varepsilon=0$, Eq. (14) will reduce to the classical simulation method of random fields. The simulated samples are periodic with the wave length $L_{0}=2 \pi / \Delta \kappa$ and period $T_{0}=2 \pi / \Delta \omega$. Furthermore, similar to the random process where spectrum is set to be zero at the origin (Shinozuka and Deodatis 1991), the following restraint must be imposed on the origin of PSD to guarantee that the sample function of the random field is ergodic in the mean and space-time correlation function (Shinozuka and Deodatis 1996)

$$
S_{f f}^{0}(0, \omega)=S_{f f}^{0}(\kappa, 0)=0 \text { for } 0 \leq \kappa<\infty \text { and } 0 \leq \omega<\infty
$$

However, this manipulation may cause significant errors when the spectrum of transformed random field has large values at zero wave number and frequency. To avoid this difficulty, the frequency shift, i.e., $\varepsilon \neq 0$, will be adopted in this study, where the restraint in Eq. (15) can be eliminated. Obviously, the simulated sample is periodic with the longer wave length $L_{0}=2 m \pi / \Delta \kappa$ and period $T_{0}=2 m \pi / \Delta \omega$. Note that Eq. (14) will reduced to the simulation formula proposed by Zerva (1992) where $\varepsilon=1 / 2$.

\subsubsection{Ergodic and statistical characteristics of sample functions}

Based on the findings in Shinozuka and Deodatis (1996), the followed statements hold: (i) the ensemble expected value and the ensemble space-time correlation function of the simulated random field are identical to corresponding targets; (ii) the spatial average and the spatial space-time correlation function based on a sample function $f^{(i)}(x, t)$ are equal to corresponding targets when the sample function $f^{(i)}(x, t)$ is simulated over the rectangular area $L_{0} \times T_{0}$. These conclusions have been also reported in Zerva (1992). 
Once the simulation of discretized random field, denoted as $\left[f\left(x_{1}, t\right), f\left(x_{2}, t\right), \cdots, f\left(x_{n}, t\right)\right]^{T}$, is performed based on Eq. (14), the realization of the corresponding multivariate homogeneous random process with the period $T_{0}$, $\left[p_{1}(t), p_{2}(t), \cdots, p_{n}(t)\right]^{T}$, can be obtained. The sample function of the homogeneous process has the following properties: (i) the ensemble expected value $E\left[p_{j}(t)\right]$ is identical to the corresponding target $E\left[p_{j}^{0}(t)\right]=0$; (ii) the ensemble auto/cross correlation function $R_{j k}(\tau)$ is identical to the corresponding target $R_{j k}^{0}(\tau)$ as $N_{1}, N_{2} \rightarrow \infty$; (iii) the temporal average of a sample function $p_{j}^{(i)}(t)$ over $T_{0}$, $\left\langle p_{j}^{(i)}(t)\right\rangle_{T_{0}}$ is identical to the corresponding target $E\left[p_{j}^{0}(t)\right]=0$; (iv) the temporal auto/cross correlation function $R_{j k}^{(\mathrm{i})}(\tau)$ over $T_{0}$ is not identical to the corresponding target $R_{j k}^{0}(\tau)$. The proofs for properties (i) and (ii) are given below while those for (iii) and (iv), i.e., ergodicity of a sample function $p_{j}^{(i)}(t)$, can be found in Appendix for brevity.

1. Show that: $E\left[p_{j}(t)\right]=E\left[p_{j}^{0}(t)\right]=0, j=1,2, \cdots, n$

Proof: $\quad E\left[p_{j}(t)\right]=E\left[p_{j}^{0}(t)\right]=0 \quad$ is $\quad$ valid because $\quad E\left[p_{j}(t)\right]=E\left[f\left(x_{j}, t\right)\right]$, $E\left[p_{j}^{0}(t)\right]=E\left[f^{0}\left(x_{j}, t\right)\right]$ and $E\left[f\left(x_{j}, t\right)\right]=E\left[f^{0}\left(x_{j}, t\right)\right]=0$.

2. Show that: $R_{j k}(\tau)=R_{j k}^{0}(\tau) j, k=1,2, \cdots, n$

Proof: Suppose $x_{j}$ and $x_{k}$ are two arbitrary points with distance $\xi_{0}$ in the space axis, i.e., $x_{k}=x_{j}+\xi_{0}$. The ensemble auto/cross correlation function between $p_{j}(t)$ and $p_{k}(t)$ can be written as:

$$
R_{j k}(\tau)=E\left[p_{j}(t) p_{k}(t+\tau)\right]=E\left[f\left(x_{j}, t\right) f\left(x_{k}, t+\tau\right)\right]=R_{f f}\left(\xi_{0}, \tau\right)
$$

Because the ensemble space-time correlation function of the simulated random field is identical to the corresponding target, one has

$$
R_{f f}\left(\xi_{0}, \tau\right)=R_{f f}^{0}\left(\xi_{0}, \tau\right)
$$


$R_{f f}^{0}\left(\xi_{0}, \tau\right)$ can be further expressed as:

$$
\begin{aligned}
R_{f f}^{0}\left(\xi_{0}, \tau\right) & =\int_{-\infty}^{\infty} \int_{-\infty}^{\infty} S_{f f}^{0}(\kappa, \omega) e^{i\left(\kappa \xi_{0}+\omega \tau\right)} d \kappa d \omega \\
& =\int_{-\infty}^{\infty}\left\{\int_{-\infty}^{\infty} S_{f f}^{0}(\kappa, \omega) e^{i \kappa \xi_{0}} d \kappa\right\} e^{i \omega \tau} d \omega \\
& =\int_{-\infty}^{\infty} S_{f}^{0}\left(\xi_{0}, \omega\right) e^{i \omega \tau} d \omega \\
& =\int_{-\infty}^{\infty} S_{j k}^{0}(\omega) e^{i \omega \tau} d \omega=R_{j k}^{0}(\tau)
\end{aligned}
$$

Hence, $R_{j k}(\tau)=R_{j k}^{0}(\tau)$ is valid. Note that in the aforementioned proof, the

XPSD between $p_{j}^{0}(t)$ and $p_{k}^{0}(t), S_{j k}^{0}(\omega)$, is identical to the one between $f^{0}\left(x_{j}, t\right)$ and $f^{0}\left(x_{k}, t\right)$.

Although the random field-based SRM cannot generate the sample function with ergodicity in terms of correlation function, the ergodicity of sample functions may lose its significance when the structural system is nonlinear or time-varying. For example, the underlying ergodicity of the input excitations cannot be taken advantage of if the ensemble averaging is conducted on usually nonergodic outputs from the analysis (Yamazaki and Shinozuka 1990). In addition, the ergodicity is lost if a Gaussian sample function is mapped to a non-Gaussian counterpart via the widely-used translation process (Bocchini and Deodatis 2008).

\subsubsection{Application of FFT algorithm}

When the simulation points are evenly distributed, two-dimensional FFT can be employed to increase the simulation efficiency. To achieve this, Eq. (14) is rewritten in the following form

$$
\begin{aligned}
f^{(i)}\left(p_{1} \Delta x, p_{2} \Delta t\right) & =\operatorname{Re}\left\{\exp \left[i \varepsilon \frac{2 \pi p_{1}}{M_{1}}+i \varepsilon \frac{2 \pi p_{2}}{M_{2}}\right] C_{q_{1} q_{2}}\right. \\
& \left.+\exp \left[i \varepsilon \frac{2 \pi p_{1}}{M_{1}}-i \varepsilon \frac{2 \pi p_{2}}{M_{2}}\right] \tilde{C}_{q_{1} q_{2}}\right\}
\end{aligned}
$$

where Re denotes the real part; $p_{1}=0,1, \ldots, M_{1}-1$ and $p_{2}=0,1, \ldots, M_{2}-1$ for

$$
\varepsilon=0 ; \quad p_{1}=0,1, \ldots, m \times M_{1}-1 \text { and } p_{2}=0,1, \ldots, m \times M_{2}-1 \text { for } \varepsilon \neq 0 ; \quad q_{1} \text { and } q_{2}
$$

are the remainder of $p_{1} / M_{1}$ and $p_{2} / M_{2}$, respectively; $C_{q_{1} q_{2}}$ and $\tilde{C}_{q_{1} q_{2}}$ are given by

$$
C_{q_{1} q_{2}}=\sum_{n_{1}=0}^{M_{1}-1} \sum_{n_{2}=0}^{M_{2}-1}\left\{B_{n_{1} n_{2}} \exp \left[i \frac{2 \pi n_{1} q_{1}}{M_{1}}+i \frac{2 \pi n_{2} q_{2}}{M_{2}}\right]\right\}
$$




$$
\tilde{C}_{q_{1} q_{2}}=\sum_{n_{1}=0}^{M_{1}-1} \sum_{n_{2}=0}^{M_{2}-1}\left\{\tilde{B}_{n_{1} n_{2}} \exp \left[i \frac{2 \pi n_{1} q_{1}}{M_{1}}-i \frac{2 \pi n_{2} q_{2}}{M_{2}}\right]\right\}
$$

where $B_{n_{1} n_{2}}$ and $\tilde{B}_{n_{1} n_{2}}$ stand for

$$
\begin{aligned}
& B_{n_{1} n_{2}}=2 \sqrt{S_{f f}^{0}\left(\kappa_{n_{1}}, \omega_{n_{2}}\right) \Delta \kappa \Delta \omega} \exp \left[i \phi_{n_{1} n_{2}}^{(1)(i)}\right] \\
& \tilde{B}_{n_{1} n_{2}}=2 \sqrt{S_{f f}^{0}\left(\kappa_{n_{1}}, \omega_{n_{2}}\right) \Delta \kappa \Delta \omega} \exp \left[i \phi_{n_{1} n_{2}}^{(2)(i)}\right]
\end{aligned}
$$

In aforementioned equations, $\Delta x$ and $\Delta t$ satisfy that $\Delta x \Delta \kappa=2 \pi / M_{1}$ and $\Delta t \Delta \omega=2 \pi / M_{2}$. In order to avoid aliasing, the following conditions must be satisfied

$$
M_{1} \geq 2 N_{1} ; \quad M_{2} \geq 2 N_{2}
$$

Note that $B_{0 n_{2}}=B_{n_{1} 0}=0$ for $n_{1}=0,1, \ldots M_{1}-1$ and $n_{2}=0,1, \ldots M_{2}-1$, and $B_{n_{1} n_{2}}=0$ for $N_{1} \leq n_{1} \leq M_{1}-1$ and/or $N_{2} \leq n_{2} \leq M_{2}-1$ must be imposed in Eq. (19) when $\varepsilon=0$.

From the simulation procedure of the random field-based method, it can be observed that the Cholesky decomposition of the spectral density matrix is not necessary. Hence, the assumption of the coherence function to follow an exponential function can be avoided which is necessary in the closed-form algorithm. Furthermore, the application of 2D FFT algorithm can expedite the computational efficiency significantly, especially for the process with a large number of simulation points.

\subsection{POD-based interpolation method}

As mentioned previously, the wind field with uniformly spaced locations can be simulated by a random field-based SRM. The wind velocities at a few unevenly spaced locations can be interpolated from their neighboring points using a POD-based technique.

Consider a part of the sample function of the aforementioned multivariate homogeneous random process $\mathbf{P}^{(i)}(t)=\left[p_{1}^{(i)}(t), p_{2}^{(i)}(t), \cdots, p_{\bar{n}}^{(i)}(t)\right]^{T}, \bar{n} \leq n . \quad \mathbf{P}^{(i)}(t)$ can be expanded on the basis of a set of optimal orthonormal bases $\boldsymbol{\Phi}=\left[\boldsymbol{\Phi}_{1}, \boldsymbol{\Phi}_{2}, \cdots, \boldsymbol{\Phi}_{\bar{n}}\right]$ and given by

$$
\mathbf{P}^{(i)}(t)=\sum_{j=1}^{\bar{n}} \mathbf{\Phi}_{j} a_{j}(t)
$$

351 where $a_{j}(t)$ is the $j$ th principal coordinate and calculated by $a_{j}(t)=\boldsymbol{\Phi}_{j}^{T} \mathbf{P}^{(i)}(t)$. In 
order to determine these bases, the projection of $\mathbf{P}^{(i)}(t)$ on them should be maximized. Typically, they can be obtained by solving the following eigenvalue equation

$$
\mathbf{R}(0) \boldsymbol{\Phi}_{j}=\lambda_{j} \boldsymbol{\Phi}_{j}
$$

where $\mathbf{R}(0)$ is the covariance matrix of the sample function $\mathbf{P}^{(i)}(t) ; \lambda_{j}$ is the $j$ th eigenvalue. Once the eigenvalues are re-ordered in the descending order, the lowest orders contain most of the energy (e.g., Kareem and Cermark 1984; Chen and Kareem 2005). This may offer guidance regarding truncation based on the energy interest in the local and global features. Accordingly, the reconstructed sample function $\hat{\mathbf{P}}^{(i)}(t)$ can be given by

$$
\hat{\mathbf{P}}^{(i)}(t)=\sum_{j=1}^{q} \boldsymbol{\Phi}_{j} a_{j}(t), q \leq \bar{n}
$$

Hereafter, POD will be used for interpolating the wind velocities for these unevenly distributed simulation points, which are not included in the sample function $\mathbf{P}^{(i)}(t)$. Once each orthonormal base $\boldsymbol{\Phi}_{j}=\left[\phi_{1 j}, \phi_{2 j}, \cdots, \phi_{\overline{n j}}\right]^{T}$ is available, the coordinates for those unevenly distributed simulation points can be interpolated and the modified base will be $\tilde{\boldsymbol{\Phi}}_{j}=\left[\phi_{1 j}, \phi_{2 j}, \cdots, \phi_{a j}, \cdots, \phi_{\overline{n j}}\right]^{T}$. Note the dimension of $\tilde{\boldsymbol{\Phi}}_{j}$ has been increased by the number of points to be interpolated. Hence, the new sample function including the interpolated points is given by

$$
\tilde{\mathbf{P}}(t)=\sum_{j=1}^{q} \tilde{\boldsymbol{\Phi}}_{j} a_{j}(t), q \leq \bar{n}
$$

From $\tilde{\mathbf{P}}(t)$, the wind velocities for interpolated points can be obtained. Finally, the whole sample function of the multivariate random process $\left[p_{1}^{0}(t), p_{2}^{0}(t), \cdots, p_{l}^{0}(t)\right]^{T}$ is achieved.

\section{Numerical example}

In order to verify the accuracy and efficiency of the proposed approach, the wind velocity field along a long-span bridge deck is employed as an example. The description of the wind velocity field will be addressed first. Then random field-based simulation results are discussed, including with and without consideration of the frequency shift. The accuracy of the random field-based simulation will be examined. Furthermore, the random field-based simulation is compared to the other methods in terms of efficiency. Finally, application of the POD-based interpolation in generating the wind velocities for those unevenly distributed points is illustrated. 


\subsection{Description of wind velocity field}

In this study, the simulation of the wind velocity field along the first kilometer-level three-pylon suspension bridge in the world, Taizhou Yangtze River Bridge, will be illustrated as an example (e.g., Chen et al. 2014). This bridge connects Taizhou and Yangzhong in Jiangsu Province of China and has a span layout of 390, 1080, 1080 and 390 m, as shown in Fig. 1.

The buffeting response of bridge deck is affected by the longitudinal and vertical turbulences simultaneously. Accordingly, the turbulences in both directions should be simulated simultaneously with the proper correlation. However, the correlation between them is generally negligible in the bridge response analysis (e.g., Chen et al. 2000). Hence, the longitudinal and vertical turbulences can be simulated individually. For the sake of brevity, only the longitudinal component of the wind velocity along the bridge deck of two main spans $(1080 \mathrm{~m}+1080 \mathrm{~m})$ will be simulated here. Note that the correlation between the longitudinal and vertical turbulences may be considered in the random field-based approach by means of the multi-dimensional random field, which will be studied in the future.

A total of 301 simulation points are evenly distributed along two main spans and thus the distance between two neighboring points is $7.2 \mathrm{~m}$. Three unevenly distributed points $\mathrm{A}, \mathrm{B}$ and $\mathrm{C}$ are employed to illustrate $\mathrm{POD}$-based interpolation, which will be discussed in section 4.4. If additional unevenly distributed points are required, the POD-based scheme can be applied to interpolate the wind velocities for these points.

Two-side Kaimal's spectrum will be chosen as the spectrum for longitudinal wind fluctuations at the height of bridge deck and is expressed as

$$
S(\omega)=\frac{200}{4 \pi} \frac{z}{U} \frac{u_{*}^{2}}{[1+50 \bar{\omega}]^{5 / 3}}
$$

where $z=65.5 \mathrm{~m}$ is the height of bridge deck; $U=38.9 \mathrm{~m} / \mathrm{s}$ is the mean wind speed on the bridge deck; $u_{*}$ is friction velocity and defined as $u_{*}=k U / \ln \left(z / z_{0}\right)$ in which $k \approx 0.4$, and $z_{0}=0.01 \mathrm{~m}$ is roughness length; $\bar{\omega}=\omega z /(2 \pi U)$.

For the coherence function between wind fluctuations at two different points $x_{j}$ and $x_{k}$ on the bridge deck, Davenport's exponential function model is adopted which can be expressed as

$$
\gamma(\xi, \omega)=\exp \left(-\frac{\lambda \xi \omega}{2 \pi U}\right)
$$

where $\lambda=7$ is decay factor; $\xi$ is the distance between $x_{j}$ and $x_{k}$. As noted in the introduction, there are some limitations concerning this exponential function model. For example, the coherence of fluctuation at very low frequency approaches unity. However, it should approach zero when the distance between two wind 
fluctuations is greater than the turbulence integral scale. In addition, the height and distance of measured points also have influence on the decay factor according to the field measurement, which is not considered in this exponential function model (ESDU 1986; Krenk 1996). To overcome these deficiencies, several non-exponential models have been introduced (e.g., Kareem 1987; Krenk 1996; Dyrbye and Hansen 1997; Mann 1998). In this study, Davenport's model is used for the sake of illustration because of its wide application in wind engineering. Also the 2D PSD of the transformed random field associated with this model has the analytical expression, as shown below. Note that other models satisfying the transformation condition in subsection 3.1 can also be utilized in the proposed method.

Correspondingly, the XPSD function between $x_{j}$ and $x_{k}$ can be given by

$$
S(\xi, \omega)=S(\omega) \exp \left(-\frac{\lambda \xi \omega}{2 \pi U}\right)
$$

As the phase angle is zero and the coherence function is even, the precondition of transformation from the multivariate homogeneous random process to space-time random field is satisfied. For a large number of locations along a bridge deck, this assumption is quite valid. According to the 2D version of the Wiener-Khintchine transform, the spatial PSD function of transformed random field can be expressed as follows:

$$
S(\kappa, \omega)=S(\omega) \frac{\alpha \omega}{\pi\left[(\alpha \omega)^{2}+\kappa^{2}\right]}
$$

where $\alpha=\lambda /(2 \pi U)$. As $\omega$ and $\kappa$ approach zero, $S(\kappa, \omega)$ becomes infinite, which means an impulse-like function exists at the origin of the spatial PSD function. However, the total energy under $S(\kappa, \omega)$, i.e., $\int_{0}^{\infty} \int_{0}^{\infty} S(\kappa, \omega) d \kappa d \omega$ is finite, i.e., 18.8 $\mathrm{m}^{2} / \mathrm{s}^{2}$. Based on Eq. (32), the corresponding space-time correlation function can be determined by Eq. (7), which is plotted in Fig. 2. This function will serve as the target in the simulation.

\subsection{Random field-based simulation results}

In the following simulation of multivariate random process, the upper cutoff frequency is selected as $4 \pi \mathrm{rad} / \mathrm{s}$ and the corresponding time interval $\Delta t$ is $0.25 \mathrm{~s}$. The discrete number of frequency $N_{2}$ is set to 2048 while the discrete numbers of wave number $N_{1}$ are chosen as 256, 512 and 1024 for comparison. It is noted that the mechanical structural properties of the long span bridge should be considered if an optimal time and space discretization is to be achieved, which may also save some computational demands (Tubino and Solari 2007). To investigate the influence of the origin of 2D PSD on simulations, both cases of $\varepsilon=0$ and $\varepsilon=1 / 2$, i.e., no 
frequency shift and frequency shift, will be considered. A total of 50 samples are used to estimate the ensemble auto/cross correlation function of the random process. Strictly, the sample function of the random process is not ergodic in sense of the correlation function of the process as shown in Appendix. However, it can be regarded to be approximately ergodic in sense of the correlation function of the process because it is ergodic in terms of random fields. Hence, the individual auto/cross correlation function is estimated based on the temporal average in order to improve the smoothness of the ensemble auto/cross correlation function.

\subsubsection{Without frequency shift}

Fig. 3 shows the space-time correlation function estimated from a sample function of the random field when $\varepsilon=0$. It can be seen that the space-time correlation function is consistent with the theoretical counterpart except some energy loss, which is caused by neglecting the origin of 2D PSD in simulation. Note that the sample function simulated from the random field are ergodic in the sense of space-time correlation function when the simulation rectangular area is equal to $L_{0} \times T_{0}$, where $L_{0}=14746 \mathrm{~m}$ for $N_{1}=1024$ and $T_{0}=1024 \mathrm{~s}$.

Fig. 4 shows the comparison of the ensemble auto/cross correlation functions estimated from the simulated sample functions of the multivariate random process and their corresponding targets. It can be seen that there exist noticeable differences between ensemble auto/cross correlation functions and their corresponding targets when $N_{1}$ is smaller. Apart from the lack of ergodicty, the main reason can be attributed to the fact that the Eq. (15) imposed on the simulation formula will result in energy loss of PSD when the spectral values in zero frequency and/or zero wave number are dominant. It is also observed that the difference between the estimated functions and targets becomes smaller with the increase of $N_{1}$ as larger $N_{1}$ leads to a smaller $\Delta \kappa$ under the prescribed cutoff wave number. When the frequency increment is fixed, the spectral value with smaller $\Delta \kappa$ will be closer to the theoretical one as compared to the counterpart with larger $\Delta \kappa$, especially for the spectral value at the origin. Accordingly, the simulation accuracy can be increased.

\subsubsection{With frequency shift}

Due to some concerns regarding the accuracy of simulation without the frequency shift, the frequency shift is adopted in this section. Fig. 5 shows the space-time correlation function estimated from a simulated sample function of the random field when $\varepsilon=1 / 2$. It can be observed that the estimated space-time correlation function matches the target (shown in Fig. 2). For the comparison with the field without frequency shift, total energy contained in 2D PSD, i.e., the value of space-time correlation function at the origin is computed. They are 15.5 and $17.3 \mathrm{~m}^{2} / \mathrm{s}^{2}$ for fields without frequency shift and with frequency shift, respectively. Clearly, the frequency shift is remarkably beneficial to increase the simulation accuracy of the random field. Therefore, $\varepsilon=1 / 2$ is used in the following discussion which leads to 
$T_{0}=2048 \mathrm{~s}$.

The simulated wind velocity time histories at point 1,2 and 76 along the bridge deck are shown in Fig. 6 ( $N_{1}=1024$ is used). It can be seen that the wind velocity of point 1 and point 2 have strong correlation. On the other hand, the correlation between point 1 and point 76 are weak. The reason is attributed to the fact that the point 1 and point 76 are more than $500 \mathrm{~m}$ apart. The comparison of the auto correlation and PSD functions for component process at point 1 with corresponding targets is illustrated in Fig. 7. It can be seen that there are still some discrepancies in the ensemble auto correlation function and PSD function when $N_{1}=512$. However, when $N_{1}$ reaches 1024, the accuracy of the simulated random process is satisfactory. The similar observation can be found in the cross correlation function, which is shown in Fig. 8.

From the aforementioned discussion, larger $N_{1}$ or $N_{2}$ or both and frequency shift can enhance the simulation accuracy of the random field and the corresponding random process. Hence, for even higher simulation accuracy, larger discrete numbers of wave number and frequency combined with a small frequency shift leads to smaller energy loss for 2D PSD. This choice leads to an extension in the period of simulated sample functions which is an insignificant issue because the simulation efficiency is very high, as shown later.

\subsection{Comparison of random field-based simulation with other methods}

To illustrate the efficiency of the proposed method, other simulation algorithms are compared. For the simulation of multivariate stationary Gaussian processes, spectral matrix should be decomposed, where Cholesky decomposition (e.g., Deodatis 1996) and closed-form formula (e.g., Yang et al. 1997) will be considered. To generate the ergodic sample function in sense of auto/cross correlation functions, the double-index technique is typically used. For comparison, the single-index counterpart is also studied. Note that the single-index technique cannot generate the ergodic sample function in sense of the cross correlation function which is widely used in the nonstationary process simulation (e.g., Huang 2014). With the combination of aforementioned techniques, four methods can be defined and referred to as Cases 1-4, as reported in Table 1. The random field-based SRM with the frequency shift $\varepsilon=1 / 2$ is referred to as Case 5 .

During the comparison, the discrete number of frequencies for all cases is set to be 1024 for expedited simulations. The number of simulation points ranges from 16 to 1024. All the algorithms are programmed in Matlab. The 64-bit computer equipped with an Intel(R) Xeon(R) E5-2609 v2 processor (2.50 GHz frequency) and $32 \mathrm{~GB}$ memory is used in the simulation.

The comparison of the computational efficiency between the five cases is shown in Table 2. Asterisk marks denote that the computational time is an estimated value. The unit of numbers is "second". Ratio 1 is defined as the ratio of the computational time of Case 2 to that of Case 5, while ratio 2 is defined as the ratio of the 
computational time of Case 4 to that of Case 5.

From the table, it can be seen that the closed-form formula can save up to almost $40 \%$ computational time compared to the Cholesky decomposition. Also, the computational efficiency of the random field-based SRM is significantly higher than that of other algorithms. Furthermore, the efficiency enhances significantly with the increase in the number of points to be simulated. For example, if 16 simulated points are considered, ratios 1 and 2 are 62.8 and 6 , respectively, where the random field-based SRM seems to be not attractive. However, as far as 128 points are concerned, ratios 1 and 2 become 16374.8 and 166.2, respectively. The computational efficiency is obviously increased which underscores the effectiveness of the proposed scheme. Clearly, the single-index technique is more meaningful than the double-index counterpart in making comparison with the random field-based SRM since the period of simulated wind fields generated by double-index technique is far greater than that via the random field-based SRM when the number of simulation points is large.

\subsection{Illustration of POD-based interpolation}

As mentioned previously, three unevenly distributed points $\mathrm{A}, \mathrm{B}$ and $\mathrm{C}$ are used for illustration. Point $\mathrm{A}$ is located at the middle of the point 75 and point 76 while points $\mathrm{B}$ and $\mathrm{C}$ are evenly distributed between point 77 and point 78 . The points from 66 to 85 will be used for the interpolation. First, the typical simulation result will be presented where the decay factor is set as 7 and linear interpolation for eigenvectors is used for simplicity. Then, the influence of the correlation of the prescribed random process and the interpolation method for eigenvectors will be investigated to demonstrate the robustness of the POD-based interpolation method.

One interpolated sample is shown in Fig. 9. It can be seen that there is a strong correlation between the wind velocity at point 76 and that at point $\mathrm{A}$. The similar phenomenon can be examined in the wind velocity of points $\mathrm{B}$ and $\mathrm{C}$ due to their short distances with point 76. Fig. 10 illustrates the comparison of estimated auto correlation function of points A and B with the prescribed ones. Fig. 11 shows the comparison of estimated cross correlation functions with targets. It can be observed that the estimated auto/cross correlation functions agree with the targets well, which verifies that the POD-based interpolation has good performance in generating wind velocities at unevenly distributed points.

As it is known, several factors may affect the POD-based interpolation effectiveness. In this study, two influencing factors including the correlation of prescribed random process and the interpolation method for eigenvectors are investigated. Fig. 12a shows results under different decay factors of coherence function. It can be seen that POD-based interpolation method performs better for lower decay factor or stronger correlation of the random process. Fig. 12b illustrates that the different methods for interpolating eigenvectors have no significant influence on interpolation results. Hence, POD-based interpolation method has a robust performance in the application.

\section{Concluding remarks}

In this paper, an efficient simulation approach was introduced for non-uniformly spaced wind velocity processes with identical auto PSD at each location. This 
approach was composed of the random field-based simulation and POD-based interpolation. First, a systematic study for the random field-based simulation for multivariate homogeneous process with evenly distributed locations was presented. By transforming the multivariate homogeneous random process to a two-dimensional homogeneous random field, the simulation of a multivariate process could be realized by that of the corresponding random field. To satisfy this transformation, the phase angle among velocities at different locations should be zero, and coherence function of random processes should be even in terms of separation distance. This requirement may not be very restrictive as the need for this technology is often experienced for a long-span bridge where all points are at the same level. Because the spectrum of the transformed random field may have a large value at zero wave number and frequency, the traditional treatment by assuming the spectrum to be zero at zero wave number and frequency may cause significant errors. Hence, a frequency shift was adopted to avoid the aforementioned problem. Although the simulated samples via random field-based approach could not satisfy ergodicity with respect to the auto and cross correlation of multivariate random process, this property may lose its meaning anyway for nonlinear or time-varying systems.

Compared to the current simulation methods, the proposed random field-based simulation has at least two advantages. First, this simulation does not require Cholesky or eigenvector decomposition of spectral matrix, which poses high computational demand in the case where a large number of points are to be simulated. Hence, the assumption of the coherence function to follow an exponential function is not necessary, which is essential for the closed-form algorithm. Second, due to the application of two-dimensional FFT, the simulation is very efficient, especially for processes with large simulation points. It is noted through numerical example that the random field-based simulation exhibited required accuracy and significantly improved efficiency. For example, the simulation efficiency could be improved by 1000 times for processes with more than 500 simulation points. Numerical example also showed that the POD-based interpolation method had a good performance to generate the wind velocities for unevenly distributed simulation points in terms of accuracy and efficiency. It should also be noted that the proposed method may introduce slight approximation of the simulated sample and the non-ergodicity from the correlation function prospective. In addition, in order to enlarge the application range of the proposed method, the multi-dimensional random field-based simulation method for random process is maybe a good choose, which will be studied in the future.

\section{Acknowledgements}

The supports by the National Science Foundation of China (Grant no. 51578471), the Applied Basic Research Program of Sichuan Province (No 2015JY0060) and the China Scholarship Council are greatly acknowledged. The third author appreciates the support by NSF (CMMI 1301008).

\section{References}

Bienkiewicz, B., Ham, H.J., and Sun, Y. (1993). "Proper orthogonal decomposition of roof pressure.” J. Wind. Eng. Ind. Aerodyn., 50, 193-202. 
Bocchini, P., and Deodatis, G. (2008). "Critical review and latest developments of a class of simulation algorithms for strongly non-Gaussian random fields." Prob. Eng. Mech., 23(4), 393-407.

Benowitz, B. A., and Deodatis, G. (2013). "Simulation of wind velocities on long span structures: A novel stochastic wave based model." Safety, Reliability, Risk and Life-Cycle Performance of Structures and Infrastructures - Proceedings of the 11th International Conference on Structural Safety and Reliability, 5549-5553.

Cao, Y., Xiang, H., and Zhou, Y. (2000). "Simulation of stochastic wind velocity field on long-span bridges.” J. Eng. Mech., 126(1), 1-6.

Carassale, L., Solari, G. (2002). "Wind modes for structural dynamics: a continuous approach.” Prob. Eng. Mech., 17(2), 157-166.

Chen, X., and Kareem, A. (2005). "Proper orthogonal decomposition-based modeling, analysis, and simulation of dynamic wind load effects on structures." J. Eng. Mech., 131(4), 1-18.

Chen, L., Letchford, C.W. (2005). "Simulation of multivariate stationary Gaussian stochastic processes: hybrid spectral representation and Proper Orthogonal Decomposition approach.” J. Eng. Mech., 131(8), 801-808.

Carassale, L., Solari, G. (2006). "Monte Carlo simulation of wind velocity fields on complex structures.” J. Wind. Eng. Ind. Aerodyn., 94(5), 323-339.

Chen, N., Li, Y., and Xiang, H. (2014). "A new simulation algorithm of multivariate short-term stochastic wind velocity field based on inverse fast Fourier transform." Eng. Struct., 80, 251-259.

Chen, X., Matsumoto, M., and Kareem, A. (2000). "Time domain flutter and buffeting response analysis of bridges." J. Eng. Mech., 126(1), 7-16.

Deodatis, G. (1996). "Simulation of ergodic multivariate stochastic processes." J. Eng. Mech., 122(8), 778-787.

Dyrbye, C., and Hansen, S.O. (1997). "Wind loads on structures." John Wiley and Sons, New York.

Di Paola, M. (1998). "Digital simulation of wind field velocity.” J. Wind. Eng. Ind. Aerodyn., 74-76, 91-109.

Di Paola, M., and Gullo, I. (2001). "Digital generation of multivariate wind field processes.” Prob. Eng. Mech., 16(1), 1-10.

Ding, Q., Zhu, L., and Xiang, H. (2011). "An efficient ergodic simulation of multivariate stochastic processes with spectral representation.” Prob. Eng. Mech., 26(2), 350-356.

ESDU. (1986). "Characteristic of atmospheric turbulence near the ground. Part III: Variations in space and time for strong winds: Neutral atmosphere." Engineering Sciences Data Unit, London.

Grigoriu, M. (2000). "A spectral representation based model for Monte Carlo simulation.” Prob. Eng. Mech., 15(4), 365-370.

Huang, G., Liao, H., and Li, M. (2013). "New Formulation of Cholesky Decomposition and Applications in Stochastic Simulation.” Prob. Eng. Mech., 34, 40-47. 
Huang, G. (2014). "An efficient simulation approach for multivariate random process: Hybrid of wavelet and spectral representation method.” Prob. Eng. Mech., 37, 74-83. Huang, G. (2015). "Application of Proper Orthogonal Decomposition in Fast Fourier Transform -Assisted Multivariate Nonstationary Process Simulation.” J. Eng. Mech., 10.1061/(ASCE)EM.1943-7889.0000923.

Kareem, A., and Cermak, J. E. (1984). "Pressure fluctuations on a square building model in boundary-layer flows.” J. Wind. Eng. Ind. Aerodyn., 16(1), 17-41.

Kareem, A. (1987). "Wind effects on structures: a probabilistic viewpoint." Prob. Eng. Mech., 2(4), 166-200.

Krenk, S. (1996). "Wind field coherence and dynamic wind forces, Proceedings of the IUTAM Symposium on Advances in nonlinear stochastic mechanics." Trondheim, Norway.

Li, Y., and Kareem, A. (1993). "Simulation of multi-variate random processes: Hybrid DFT and digital filtering approach.” J. Eng. Mech., 119(5), 1078-1098.

Li, Y., and Kareem, A. (1995). "Stochastic decomposition and application to probabilistic dynamics.” J. Eng. Mech., 121(1), 162-174.

Liu, Z., Chen, J., and Li, L. (2011). "Orthogonal expansion of Gaussian wind velocity field and PDEM-based vibration analysis of wind-excited structures." J. Wind. Eng. Ind. Aerodyn., 99, 1207-1220.

Mann, J. (1998). "Wind field simulation.” Prob. Eng. Mech., 13(4), 269-282.

Shinozuka, M., (1972). "Monte Carlo solution of structural dynamics." Comput Struct., 2(5), 855-874.

Shinozuka, M., and Jan, C. (1972). "Digital simulation of random processes and its applications.” J. Sound Vib., 25(1), 111-128.

Spanos, P D., and Mignolet, M.P. (1989). "ARMA Monte Carlo simulation in probabilistic structural analysis." Shock. Vib. Dig., 21(11), 3-14.

Shinozuka, M., and Deodatis, G. (1991). "Simulation of stochastic processes by spectral representation.” Appl. Mech. Rev., 44(4), 191-204.

Shinozuka, M., and Deodatis, G (1996). "Simulation of multi-dimensional Gaussian stochastic fields by spectral representation.” Appl Mech Rev., 49(1), 29-53.

Tamura, Y., Suganuma, S., Kikuchi, H., and Hibi, K. (1999). "Proper orthogonal decomposition of random wind pressure field.” J. Fluids Struct., 13, 1069-1095.

Tubino, F., and Solari, G. (2005). "Double POD for representing and simulating turbulence fields.” J. Eng. Mech., 131(12), 1302-1312.

Tubino, F., and Solari, G. (2007). "Gust buffeting of long span bridges: Double Modal Transformation and effective turbulence.” Eng. Struct., 29(8), 1698-1707. Yang, J.N. (1972). "Simulation of random envelope processes." J. Sound Vib., 25, 73-85.

Yamazaki, F., and Shinozuka, M. (1990). "Simulation of stochastic fields by statistical preconditioning.” J. Eng. Mech., 116(2), 268-287.

Yang, W.W., Chang, T., and Chang, C.C. (1997). "An efficient wind field simulation technique for bridges.” J. Wind. Eng. Ind. Aerodyn., 67, 697-708.

Zerva, A. (1992). "Seismic ground motion simulations from a class of spatial variability models.” Earthquake. Eng. Struct. Dyn., 21(4), 351-361. 
Zerva, A., Zervas, V. (2002). "Spatial variation of seismic ground motions: An overview." Appl. Mech. Rev., 55(3), 271-297.

\section{Appendix}

In this appendix, the ergodicity of a sample function of the multivariate homogeneous random process generated by the random field-based SRM will be addressed. Suppose that the length of the simulated sample, $T$, is equal to the period, $T_{0}$, which varies with the selected value of $\varepsilon$ and is expressed as

$$
T_{0}= \begin{cases}2 \pi / \Delta \omega & \varepsilon=0 \\ 2 \pi m / \Delta \omega & \varepsilon \neq 0\end{cases}
$$

1), Show that: $\left\langle p_{j}^{(i)}(t)\right\rangle_{T}=E\left[p_{j}^{0}(t)\right]=0$ when $T=T_{0}$

Proof: The temporal average of a sample function $p_{j}^{(i)}(t)$ over a time interval $T=T_{0},\left\langle p_{j}^{(i)}(t)\right\rangle_{T}$, can be written as:

$$
\left\langle p_{j}^{(i)}(t)\right\rangle_{T}=\frac{1}{T} \int_{0}^{T} p_{j}^{(i)}(t) d t=\frac{1}{T} \int_{0}^{T} f^{(i)}\left(x_{j}, t\right) d t
$$

$=\frac{1}{T} \int_{0}^{T} \sqrt{2} \sum_{n_{1}=0}^{N_{1}-1} \sum_{n_{2}=0}^{N_{2}-1}\left\{A_{n_{1} n_{2}}\left[\cos \left(\kappa_{n_{1}} x_{j}+\omega_{n_{2}} t+\phi_{n_{1} n_{2}}^{(1)(i)}\right)+\cos \left(\kappa_{n_{1}} x_{j}-\omega_{n_{2}} t+\phi_{n_{1} n_{2}}^{(2)(i)}\right)\right]\right\} d t$

$$
=\frac{\sqrt{2}}{T} \sum_{n_{1}=0}^{N_{1}-1} \sum_{n_{2}=0}^{N_{2}-1}\left\{A_{n_{1} n_{2}}\left[\int_{0}^{T} \cos \left(\kappa_{n_{1}} x_{j}+\omega_{n_{2}} t+\phi_{n_{1} n_{2}}^{(1)(i)}\right) d t+\int_{0}^{T} \cos \left(\kappa_{n_{1}} x_{j}-\omega_{n_{2}} t+\phi_{n_{1} n_{2}}^{(2)(i)}\right) d t\right]\right\}
$$

where $A_{n_{1} n_{2}}=\sqrt{2 S_{f f}^{0}\left(\kappa_{n_{1}}, \omega_{n_{2}}\right) \Delta \kappa \Delta \omega} ; \kappa_{n_{1}}=\left(n_{1}+\varepsilon\right) \Delta \kappa ; \omega_{n_{2}}=\left(n_{2}+\varepsilon\right) \Delta \omega ; \varepsilon$ is a fraction $1 / m$ ( $m$ is integer larger than 1$)$; integrands $\cos \left(\kappa_{n_{1}} x_{j}+\omega_{n_{2}} t+\phi_{n_{1} n_{2}}^{(1)(i)}\right)$ and $\cos \left(\kappa_{n_{1}} x_{j}-\omega_{n_{2}} t+\phi_{n_{1} n_{2}}^{(2)(i)}\right)$ are both periodic functions of $t$ with a period equal to $T_{0}$ when $n_{2} \neq 0$. While $n_{2}=0$, the above integrands will reduce to some constants.

Taking also into account that $A_{n_{1} 0}=0$ for $n_{1}=0,1, \ldots, N_{1}-1$, it is obvious that:

$$
\left\langle p_{j}^{(i)}(t)\right\rangle_{T}=0 \text { when } T=T_{0}
$$

2), Show that: $R_{j k}^{(i)}(\tau)=\left\langle p_{j}^{(i)}(t) p_{k}^{(i)}(t+\tau)\right\rangle_{T} \neq R_{j k}^{0}(\tau)$ when $T=T_{0}$

Proof: The temporal auto/cross correlation function of sample function $f_{j}^{(i)}(t)$ and $f_{k}^{(i)}(t)$ with a time interval equal to $T_{0}$ can be written as: 


$$
\begin{aligned}
& R_{j k}^{(i)}(\tau)=\left\langle p_{j}^{(i)}(t) p_{k}^{(i)}(t+\tau)\right\rangle_{T} \\
& =\frac{1}{T} \int_{0}^{T} p_{j}^{(i)}(t) p_{k}^{(i)}(t+\tau) d t=\frac{1}{T} \int_{0}^{T} f^{(i)}\left(x_{j}, t\right) f^{(i)}\left(x_{k}, t+\tau\right) d t \\
& =\frac{2}{T} \int_{0}^{T} \sum_{n_{1}=0}^{N_{1}-1} \sum_{n_{2}=0}^{N_{2}-1}\left\{A_{n_{1} n_{2}}\left[\cos \left(\kappa_{n_{1}} x_{j}+\omega_{n_{2}} t+\phi_{n_{1} n_{2}}^{(1)(i)}\right)+\cos \left(\kappa_{n_{1}} x_{j}-\omega_{n_{2}} t+\phi_{n_{1} n_{2}}^{(2)(i)}\right)\right]\right\} \\
& \cdot \sum_{m_{1}=0}^{N_{1}-1} \sum_{m_{2}=0}^{N_{2}-1}\left\{A_{m_{1} m_{2}}\left[\cos \left(\kappa_{m_{1}} x_{k}+\omega_{m_{2}}(t+\tau)+\phi_{m_{1} m_{2}}^{(1)(i)}\right)+\cos \left(\kappa_{m_{1}} x_{k}-\omega_{m_{2}}(t+\tau)+\phi_{m_{1} m_{2}}^{(2)(i)}\right)\right]\right\} d t \\
& =\frac{2}{T} \int_{0}^{T} \sum_{n_{1}=0}^{N_{1}-1} \sum_{n_{2}=0}^{N_{2}-1} \sum_{m_{1}=0}^{N_{1}-1} \sum_{m_{2}=0}^{N_{2}-1} A_{n_{1} n_{2}} A_{m_{1} m_{2}}\left[\cos \left(\kappa_{n_{1}} x_{j}+\omega_{n_{2}} t+\phi_{n_{1} n_{2}}^{(1)(i)}\right)+\cos \left(\kappa_{n_{1}} x_{j}-\omega_{n_{2}} t+\phi_{n_{1} n_{2}}^{(2)(i)}\right)\right] \\
& \cdot\left[\cos \left(\kappa_{m_{1}} x_{k}+\omega_{m_{2}}(t+\tau)+\phi_{m_{1} m_{2}}^{(1)(i)}\right)+\cos \left(\kappa_{m_{1}} x_{k}-\omega_{m_{2}}(t+\tau)+\phi_{m_{1} m_{2}}^{(2)(i)}\right)\right] d t \\
& =\frac{1}{T} \sum_{n_{1}=0}^{N_{1}-1} \sum_{n_{2}=0}^{N_{2}-1} \sum_{m_{1}=0}^{N_{1}-1} \sum_{m_{2}=0}^{N_{2}-1} A_{n_{1} n_{2}} A_{m_{1} m_{2}}\left\{\int_{0}^{T} \cos \left(\kappa_{n_{1}} x_{j}+\kappa_{m_{1}} x_{k}+\omega_{n_{2}} t+\omega_{m_{2}}(t+\tau)+\phi_{n_{1} n_{2}}^{(1)(i)}+\phi_{m_{1} m_{2}}^{(1)(i)}\right) d t\right. \\
& \left.+\int_{0}^{T} \cos \left(\kappa_{n_{1}} x_{j}-\kappa_{m_{1}} x_{k}+\omega_{n_{2}} t-\omega_{m_{2}}(t+\tau)+\phi_{n_{1} n_{2}}^{(1)(i)}-\phi_{m_{1} m_{2}}^{(1)(i)}\right) d t\right\} \\
& +\frac{1}{T} \sum_{n_{1}=0}^{N_{1}-1} \sum_{n_{2}=0}^{N_{2}-1} \sum_{m_{1}=0}^{N_{1}-1} \sum_{m_{2}=0}^{N_{2}-1} A_{n_{1} n_{2}} A_{m_{1} m_{2}}\left\{\int_{0}^{T} \cos \left(\kappa_{n_{1}} x_{j}+\kappa_{m_{1}} x_{k}-\omega_{n_{2}} t+\omega_{m_{2}}(t+\tau)+\phi_{n_{1} n_{2}}^{(2)(i)}+\phi_{m_{1} m_{2}}^{(1)(i)}\right) d t\right. \\
& \left.+\int_{0}^{T} \cos \left(\kappa_{n_{1}} x_{j}-\kappa_{m_{1}} x_{k}-\omega_{n_{2}} t-\omega_{m_{2}}(t+\tau)+\phi_{n_{1} n_{2}}^{(2)(i)}-\phi_{m_{1} m_{2}}^{(1)(i)}\right) d t\right\} \\
& +\frac{1}{T} \sum_{n_{1}=0}^{N_{1}-1} \sum_{n_{2}=0}^{N_{2}-1} \sum_{m_{1}=0}^{N_{1}-1} \sum_{m_{2}=0}^{N_{2}-1} A_{n_{1} n_{2}} A_{m_{1} m_{2}}\left\{\int_{0}^{T} \cos \left(\kappa_{n_{1}} x_{j}+\kappa_{m_{1}} x_{k}+\omega_{n_{2}} t-\omega_{m_{2}}(t+\tau)+\phi_{n_{1} n_{2}}^{(1)(i)}+\phi_{m_{1} m_{2}}^{(2)(i)}\right) d t\right. \\
& \left.+\int_{0}^{T} \cos \left(\kappa_{n_{1}} x_{j}-\kappa_{m_{1}} x_{k}+\omega_{n_{2}} t+\omega_{m_{2}}(t+\tau)+\phi_{n_{1} n_{2}}^{(1)(i)}-\phi_{m_{1} m_{2}}^{(2)(i)}\right) d t\right\} \\
& +\frac{1}{T} \sum_{n_{1}=0}^{N_{1}-1} \sum_{n_{2}=0}^{N_{2}-1} \sum_{m_{1}=0}^{N_{1}-1} \sum_{m_{2}=0}^{N_{2}-1} A_{n_{1} n_{2}} A_{m_{1} m_{2}}\left\{\int_{0}^{T} \cos \left(\kappa_{n_{1}} x_{j}+\kappa_{m_{1}} x_{k}-\omega_{n_{2}} t-\omega_{m_{2}}(t+\tau)+\phi_{n_{1} n_{2}}^{(2)(i)}+\phi_{m_{1} m_{2}}^{(2)(i)}\right) d t\right. \\
& \left.+\int_{0}^{T} \cos \left(\kappa_{n_{1}} x_{j}-\kappa_{m_{1}} x_{k}-\omega_{n_{2}} t+\omega_{m_{2}}(t+\tau)+\phi_{n_{1} n_{2}}^{(2)(i)}-\phi_{m_{1} m_{2}}^{(2)(i)}\right) d t\right\}
\end{aligned}
$$

Considering the following two cases, the eight integrands in the aforementioned equation can be simplified. When $n_{2} \neq m_{2}$, it is straightforward to show that eight

731 integrands are equal to zero under the case of $T=T_{0}$. For example, the third integrand

$$
I_{3}=\cos \left(\kappa_{n_{1}} x_{j}+\kappa_{m_{1}} x_{k}-\omega_{n_{2}} t+\omega_{m_{2}}(t+\tau)+\phi_{n_{1} n_{2}}^{(2)(i)}+\phi_{m_{1} m_{2}}^{(1)(i)}\right)
$$

734 is a periodic function of $t$ with a period equal to $T_{0} /\left|n_{2}-m_{2}\right|, n_{2}=0,1, \ldots, N_{2}-1$ and $m_{2}=0,1, \ldots, N_{2}-1$. It is therefore obvious that the following equation is valid. 
Similarly, it can be shown that the remaining seven integrands are also equal to zero when $T=T_{0}$.

Consequently, the only non-zero terms in the expression for $R_{j k}^{(i)}(\tau)$ will remain for $n_{2}=m_{2}$. Accordingly, Eq. (36) reduces to

$$
\begin{aligned}
& R_{j k}^{(i)}(\tau)=\frac{1}{T} \sum_{n_{1}=0}^{N_{1}-1} \sum_{n_{2}=0}^{N_{2}-1} \sum_{m_{1}=0}^{N_{1}-1} A_{n_{1} n_{2}} A_{m_{1} n_{2}}\left\{\int_{0}^{T} \cos \left(\kappa_{n_{1}} x_{j}+\kappa_{m_{1}} x_{k}+\omega_{n_{2}} t+\omega_{n_{2}}(t+\tau)+\phi_{n_{1} n_{2}}^{(1)(i)}+\phi_{m_{1} n_{2}}^{(1)(i)}\right) d t\right. \\
& \left.+\int_{0}^{T} \cos \left(\kappa_{n_{1}} x_{j}-\kappa_{m_{1}} x_{k}+\omega_{n_{2}} t-\omega_{n_{2}}(t+\tau)+\phi_{n_{1} n_{2}}^{(1)(i)}-\phi_{m_{1} n_{2}}^{(1)(i)}\right) d t\right\} \\
& +\frac{1}{T} \sum_{n_{1}=0}^{N_{1}-1} \sum_{n_{2}=0}^{N_{2}-1} \sum_{m_{1}=0}^{N_{1}-1} A_{n_{1} n_{2}} A_{m_{1} n_{2}}\left\{\int_{0}^{T} \cos \left(\kappa_{n_{1}} x_{j}+\kappa_{m_{1}} x_{k}-\omega_{n_{2}} t+\omega_{n_{2}}(t+\tau)+\phi_{n_{1} n_{2}}^{(2)(i)}+\phi_{m_{1} n_{2}}^{(1)(i)}\right) d t\right. \\
& \left.+\int_{0}^{T} \cos \left(\kappa_{n_{1}} x_{j}-\kappa_{m_{1}} x_{k}-\omega_{n_{2}} t-\omega_{n_{2}}(t+\tau)+\phi_{n_{1} n_{2}}^{(2)(i)}-\phi_{m_{1} n_{2}}^{(1)(i)}\right) d t\right\} \\
& +\frac{1}{T} \sum_{n_{1}=0}^{N_{1}-1} \sum_{n_{2}=0}^{N_{2}-1} \sum_{m_{1}=0}^{N_{1}-1} A_{n_{1} n_{2}} A_{m_{1} n_{2}}\left\{\int_{0}^{T} \cos \left(\kappa_{n_{1}} x_{j}+\kappa_{m_{1}} x_{k}+\omega_{n_{2}} t-\omega_{n_{2}}(t+\tau)+\phi_{n_{1} n_{2}}^{(1)(i)}+\phi_{m_{1} n_{2}}^{(2)(i)}\right) d t\right. \\
& \left.+\int_{0}^{T} \cos \left(\kappa_{n_{1}} x_{j}-\kappa_{m_{1}} x_{k}+\omega_{n_{2}} t+\omega_{n_{2}}(t+\tau)+\phi_{n_{1} n_{2}}^{(1)(i)}-\phi_{m_{1} n_{2}}^{(2)(i)}\right) d t\right\} \\
& +\frac{1}{T} \sum_{n_{1}=0}^{N_{1}-1} \sum_{n_{2}=0}^{N_{2}-1} \sum_{m_{1}=0}^{N_{1}-1} A_{n_{1} n_{2}} A_{m_{1} n_{2}}\left\{\int_{0}^{T} \cos \left(\kappa_{n_{1}} x_{j}+\kappa_{m_{1}} x_{k}-\omega_{n_{2}} t-\omega_{n_{2}}(t+\tau)+\phi_{n_{1} n_{2}}^{(2)(i)}+\phi_{m_{1} n_{2}}^{(2)(i)}\right) d t\right. \\
& \left.+\int_{0}^{T} \cos \left(\kappa_{n_{1}} x_{j}-\kappa_{m_{1}} x_{k}-\omega_{n_{2}} t+\omega_{n_{2}}(t+\tau)+\phi_{n_{1} n_{2}}^{(2)(i)}-\phi_{m_{1} n_{2}}^{(2)(i)}\right) d t\right\}
\end{aligned}
$$

When $T=T_{0}$, the temporal auto/cross correlation function can be expressed as:

$$
\begin{aligned}
R_{j k}^{(i)}(\tau)=\sum_{n_{1}=0}^{N_{1}-1} \sum_{n_{2}=0}^{N_{2}-1} \sum_{m_{1}=0}^{N_{1}-1} A_{n_{1} n_{2}} A_{m_{1} n_{2}} & {\left[\cos \left(\kappa_{n_{1}} x_{j}-\kappa_{m_{1}} x_{k}-\omega_{n_{2}} \tau+\phi_{n_{1} n_{2}}^{(1)(i)}-\phi_{m_{1} n_{2}}^{(1)(i)}\right)\right.} \\
+ & \cos \left(\kappa_{n_{1}} x_{j}+\kappa_{m_{1}} x_{k}+\omega_{n_{2}} \tau+\phi_{n_{1} n_{2}}^{(2)(i)}+\phi_{m_{1} n_{2}}^{(1)(i)}\right) \\
+ & \cos \left(\kappa_{n_{1}} x_{j}+\kappa_{m_{1}} x_{k}-\omega_{n_{2}} \tau+\phi_{n_{1} n_{2}}^{(1)(i)}+\phi_{m_{1} n_{2}}^{(2)(i)}\right) \\
+ & \left.\cos \left(\kappa_{n_{1}} x_{j}-\kappa_{m_{1}} x_{k}+\omega_{n_{2}} \tau+\phi_{n_{1} n_{2}}^{(2)(i)}-\phi_{m_{1} n_{2}}^{(2)(i)}\right)\right]
\end{aligned}
$$

Note that the ensemble auto/cross correlation $R_{j k}(\tau)$ of the simulated stochastic process admits the following form (Shinozuka and Deodatis 1996)

$$
R_{j k}(\tau)=R_{f f}\left(\xi_{0}, \tau\right)=\sum_{n_{1}=0}^{N_{1}-1} \sum_{n_{2}=0}^{N_{2}-1}\left\{A_{n_{1} n_{2}}^{2}\left[\cos \left(\kappa_{n_{1}} \xi_{0}+\omega_{n_{2}} \tau\right)+\cos \left(\kappa_{n_{1}} \xi_{0}-\omega_{n_{2}} \tau\right)\right]\right\}
$$

Therefore, $R_{j k}^{(i)}(\tau) \neq R_{j k}^{0}(\tau)$ 


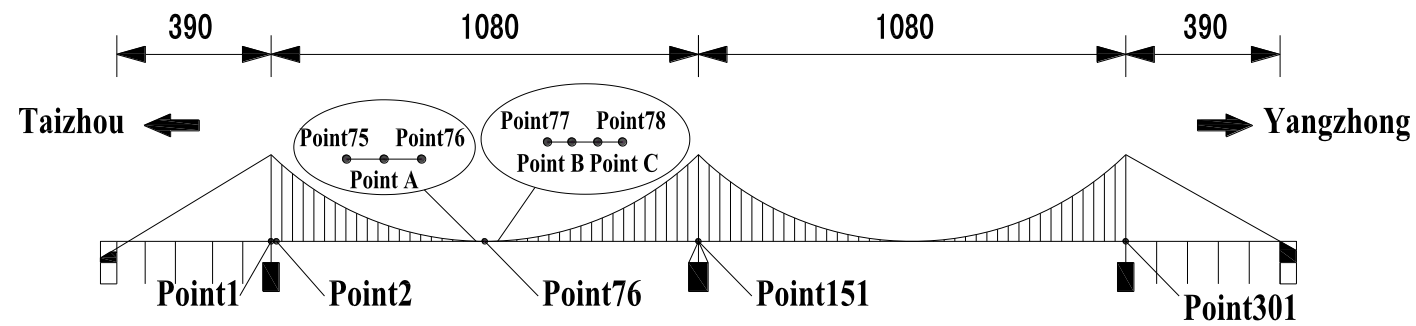

Fig. 1 Simulated points distributed on the bridge deck 


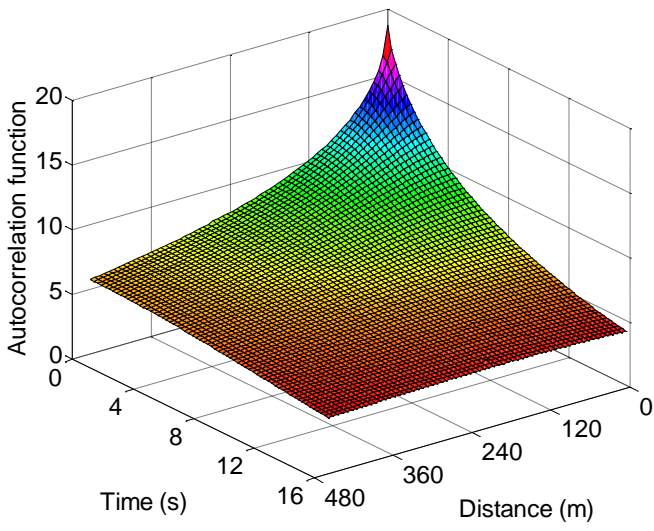

3

Fig. 2 Theoretical space-time correlation function

4 


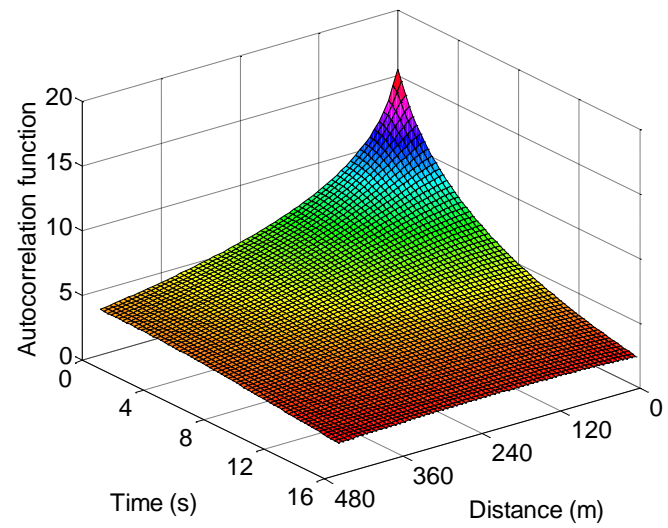

3

Fig. 3 Space-time correlation function without frequency shift $\left(N_{1}=1024\right)$ 


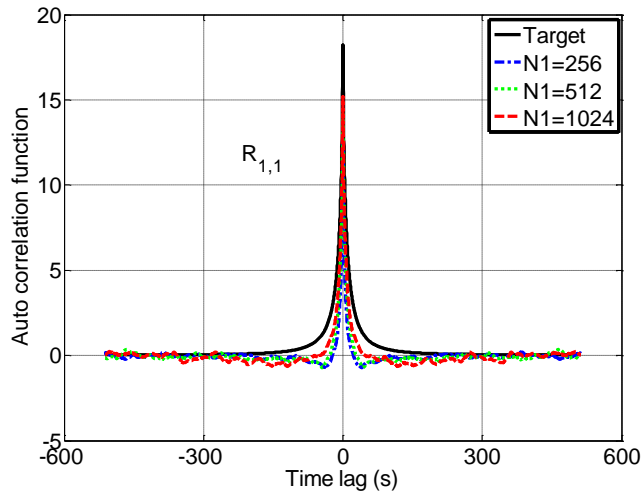

(a) At point 1

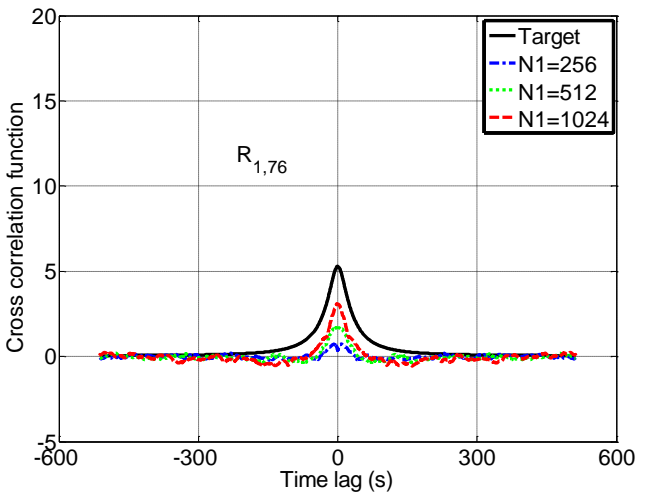

(b) Between points 1 and 76

Fig. 4 Comparison of auto/cross correlation functions (without frequency shift) 5 


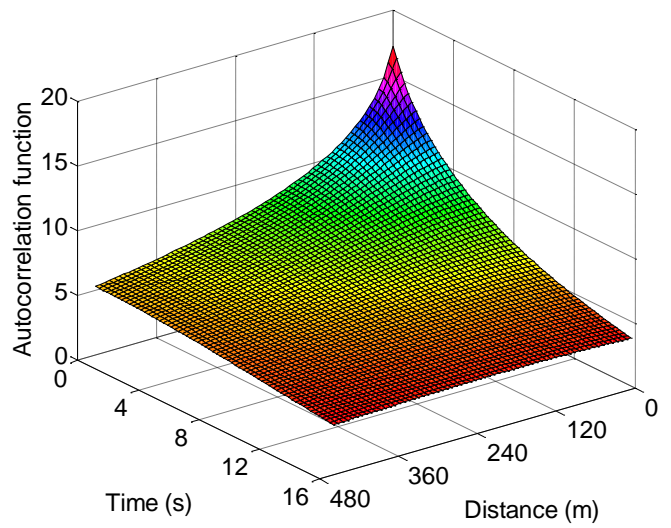

3

Fig. 5 Space-time correlation function with frequency shift $\left(N_{1}=1024\right)$

4 


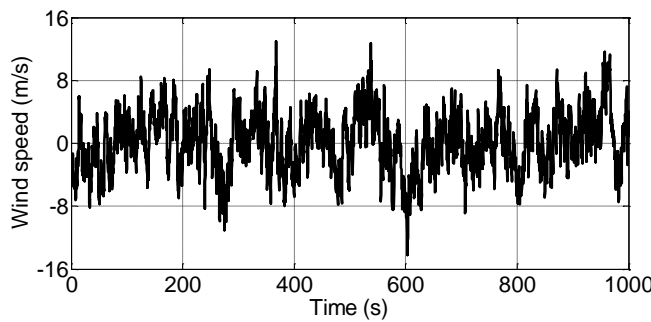

(a) Point 1

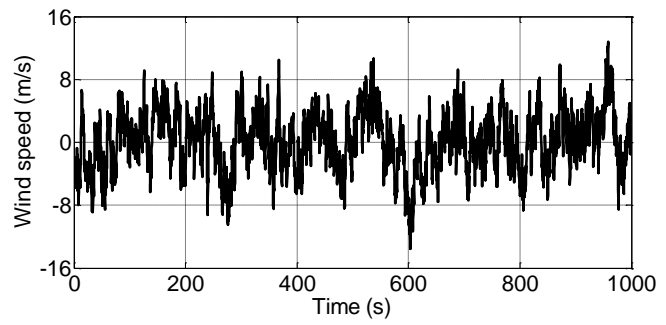

(b) Point 2
4

5

6

7

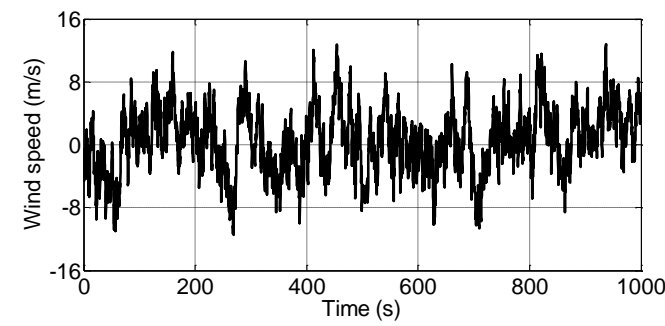

(c) Point 76

Fig. 6 Simulated wind velocities $\left(N_{1}=1024\right)$ 


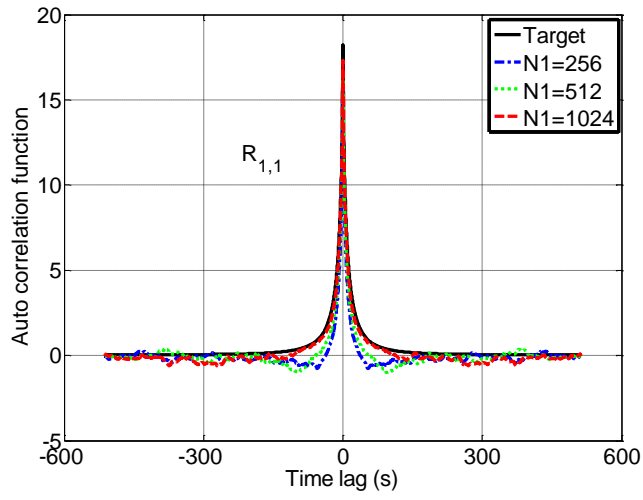

(a) Auto correlation function

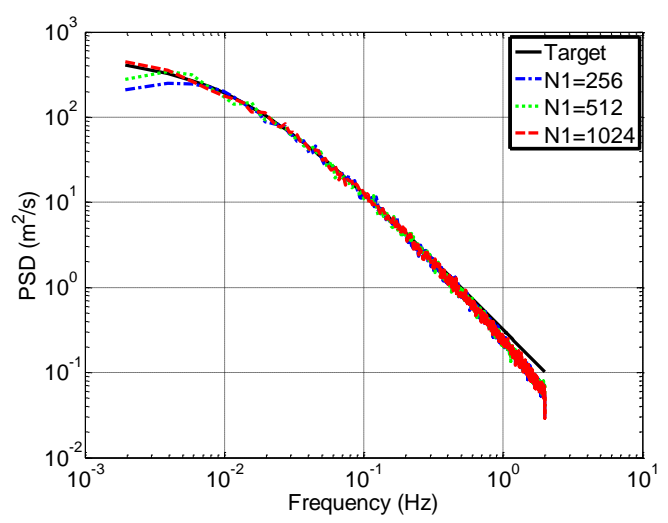

(b) PSD function

Fig. 7 Comparison of auto correlation and PSD functions at point 1 (with frequency shift) 


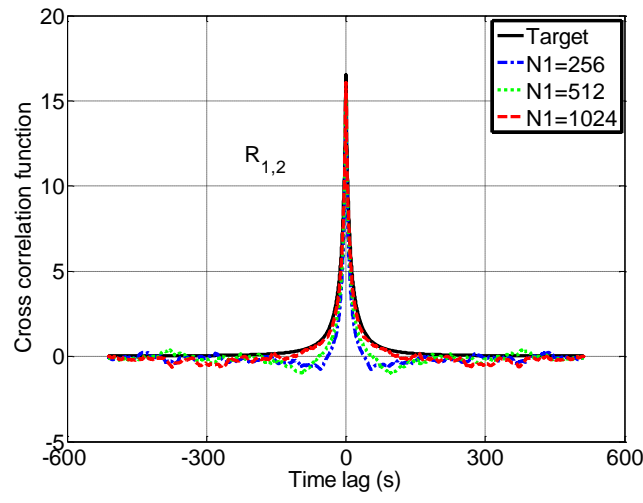

(a) Between points 1 and 2

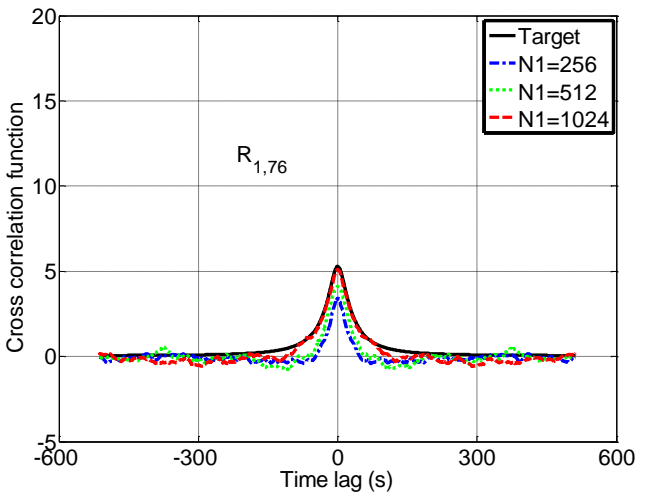

(b) Between points 1 and 76

Fig. 8 Comparison of cross correlation functions (with frequency shift) 


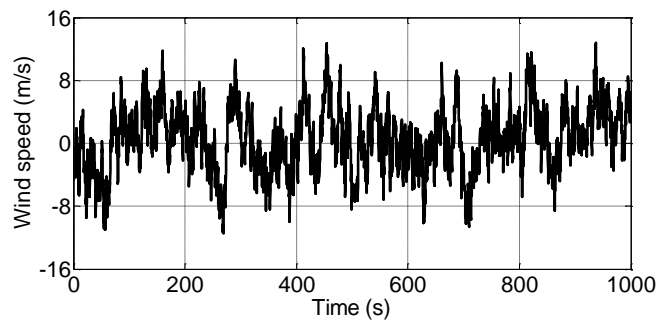

(a) Point A

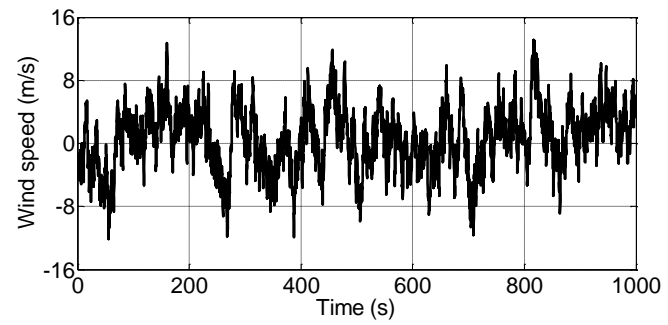

(b) Point B
4

5

6

7

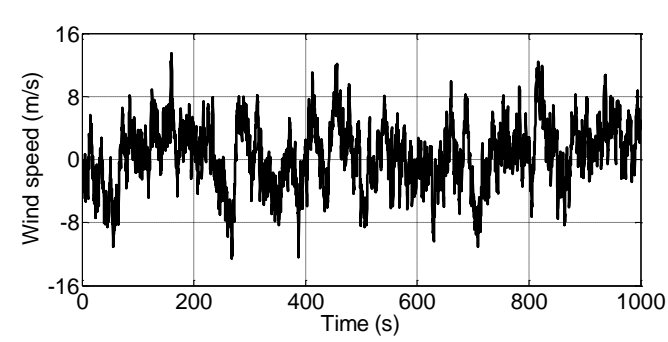

(c) Point C

Fig. 9 Interpolated sample of wind speed 


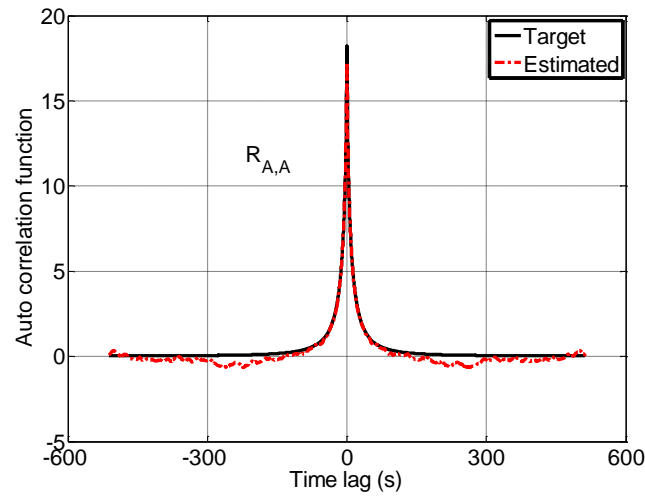

(a) Point $\mathrm{A}$

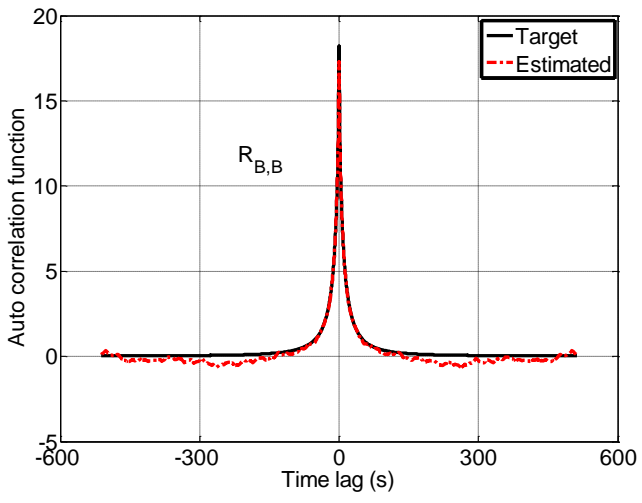

(b) Point B

Fig. 10 Comparison of auto correlation functions for points $\mathrm{A}$ and $\mathrm{B}$ 


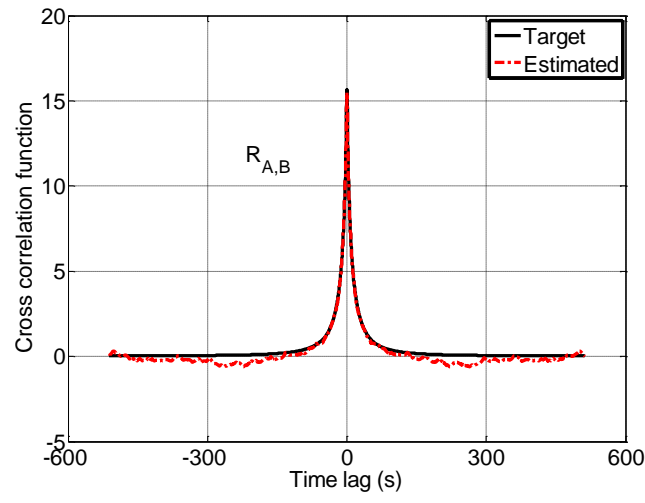

(a) Between points A and B

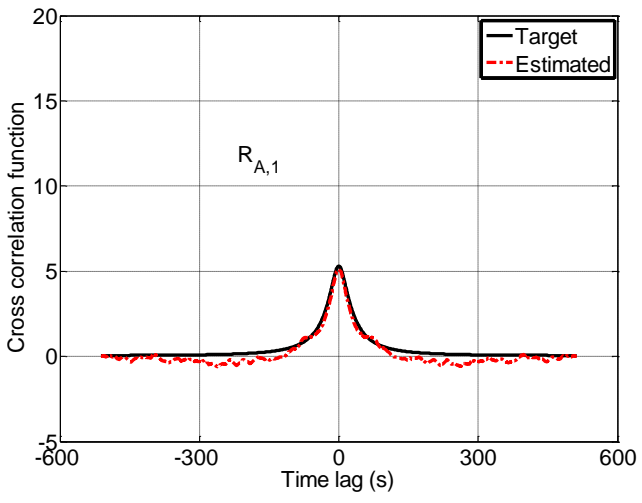

(b) Between points A and 1

Fig. 11 Comparison of cross correlation functions associated with point $A$ 


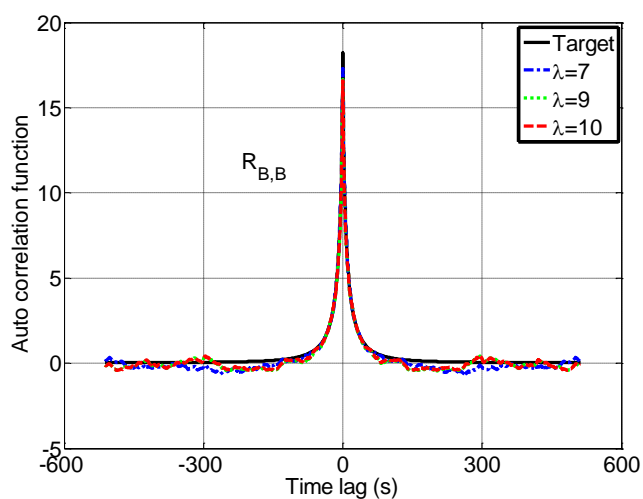

(a) Decay factor of random process

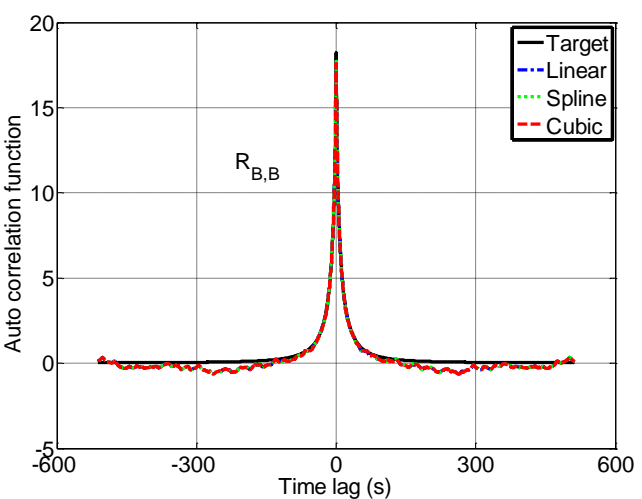

(b) Interpolation method for eigenvectors Fig. 12 Influence of decay factor and interpolation method (Point B) 
Table 1 Definition of four methods

\begin{tabular}{ccc}
\hline Types & double index & single index \\
\hline $\begin{array}{c}\text { Cholesky decomposition } \\
\text { closed-form formula }\end{array}$ & Case 1 & Case 3 \\
\hline
\end{tabular}

3

4 
Table 2 Comparison of computational efficiency

\begin{tabular}{cccccccc}
\hline & \multicolumn{7}{c}{ Simulated points } \\
\cline { 2 - 8 } & 16 & 32 & 64 & 128 & 256 & 512 & 1024 \\
\hline Case 1 & 4.04 & 28.59 & 225.27 & 2290.90 & $2.2 \mathrm{e} 4^{*}$ & $1.8 \mathrm{e} 5^{*}$ & $1.4 \mathrm{e} 6^{*}$ \\
Case 2 & 2.51 & 18.34 & 140.31 & 1473.73 & $1.1 \mathrm{e} 4^{*}$ & $7.2 \mathrm{e} 4^{*}$ & $5.4 \mathrm{e} 5^{*}$ \\
Case 3 & 0.34 & 1.26 & 4.96 & 20.04 & 84.55 & 382.78 & 1720.95 \\
Case 4 & 0.24 & 0.88 & 3.49 & 14.96 & 61.95 & 279.81 & 1205.87 \\
Case 5 & 0.04 & 0.04 & 0.06 & 0.09 & 0.16 & 0.28 & 0.56 \\
Ratio 1 & 62.8 & 458.5 & 2338.5 & 16374.8 & $6.8 \mathrm{e} 4^{*}$ & $2.5 \mathrm{e} 5^{*}$ & $9.6 \mathrm{e} 5^{*}$ \\
Ratio 2 & 6 & 22 & 58.2 & 166.2 & 387.2 & 999.3 & 2153.3 \\
\hline
\end{tabular}

2 Note: $*$ denotes the estimated value

3

4 